\title{
Research for the Detecting Method of Concrete Structure Internal Defects with Spherical Embedded Ultrasonic Transducer
}

\author{
Xingbin Zhang, Sha Fang*, Hua Rong, Jingshu Wu, Guanqiao Zhao \\ National Test Center of Quality and Safety Supervision for Industrial Buildings and Structures, Central Research Institute of Building and \\ Construction Co., Ltd, MCC Group, Beijing, China
}

Email address:

fangsha0618@163.com (Sha Fang), re-zhang@126.com (Xingbin Zhang)

${ }^{*}$ Corresponding author

\section{To cite this article:}

Xingbin Zhang, Sha Fang, Hua Rong, Jingshu Wu, Guanqiao Zhao. Research for the Detecting Method of Concrete Structure Internal Defects with Spherical Embedded Ultrasonic Transducer. American Journal of Civil Engineering. Vol. 9, No. 1, 2021, pp. 11-17.

doi: 10.11648/j.ajce.20210901.12

Received: December 20, 2018; Accepted: February 25, 2019; Published: February 10, 2021

\begin{abstract}
As a nondestructive detecting method, ultrasonic detection has been widely adopted in concrete structure detections. This approach would not damage the structural integrity, and could fulfill most of the detecting requirements of various concrete structures. However, for mass concrete and complicated concrete structural elements, there are still many problems of detection to be solved, such as the operating position of detection could not be achieved easily for technicians, especially in some narrow spaces, or the volume of elements might be quite large so that the detecting distance is far beyond the effective detecting distance of traditional transducers. After researching the technical performances of traditional ultrasonic transducers and ultrasonic detecting method, a brand new type of ultrasonic transducer adopted spherical piezoelectric ceramic chips has been developed. The transducer could be embedded inside concrete structure, and motivate or receive ultrasonic signal in all directions pair with external or internal transducers. Testing with the embedded transducers would not be limited by on-site conditions or other enviorenmental conditions. The transducer could be used in realtime systems for monitoring or detecting internal compactness situation of concrete elements. The technical characteristics of spherical transducer have been verified by a series of experiments in laboratory. Moreover, the detecting method with spherical transducer has been proposed, which could be used in the inspection for mass concrete and complicated structural concrete elements. Meanwhile, the appropriate technical schemes for reactor containment of nuclear power plant have been designed and has already been applied in nuclear power plant.
\end{abstract}

Keywords: Concrete, Rock Slope, Spherical Ultrasonic Transducer, Defect Detection

\section{Introduction}

Various structural modes such as mass concrete structures, steel plate-concrete (SC) structures, permanent formwork concrete structures and other types have been adopted in the nuclear power plants under construction or to be built in China. The potential defects inside structure of these structural types cannot be discovered easily by surface inspection only. For instance, pouring problems of mass concrete structure occur during construction in both of the reactor types of CPR 1000 and EPR. Particularly, as the outermost layer of nuclear safety barriers, the compactness of reactor containment concrete is significantly important, and shall be highly concerned. AP1000 nuclear power units will be adopted as the main reactor type in China in the future. The modular construction technique is adopted in AP1000. SC structure with self-compacting concrete (SCC) is widely used in AP1000 units [1]. Voids and cavities are easily observed in some areas especially like corners due to the special and complicated structure. These defects would impact the lifetime of structure. Consequently, the concrete structure safety judgment is proved to be of uppermost priority.

To overcome the limitations of traditional ultrasonic transducers and detecting methods when utilized in mass 
concrete structures or complicated structural elements, a brand new spherical embedded ultrasonic transducer has been invented and produced. The detecting methods and schemes for the structures mentioned above have been developed as well. With the new transducers and methods, the detection of internal defects could be easily and conveniently performed. The spherical emedded ultrasonic transducer and detecting method could be adopted in many construction areas, such as nuclear power plant, bridge, dam, tunnel, etc.

\section{Nondestructive Testing Methods}

\subsection{Ultrasonic Detection}

Ultrasonic nondestructive detecting approach has been constantly adopted in nondestructive detection. The method is based on ultrasonic waves. An ultrasonic transducer is motivated by a pulse produced by the instrument and generates ultrasonic waves. The waves propagate into the structure and are received by another transducer on the external surface of the structure (Figure 1) [2].

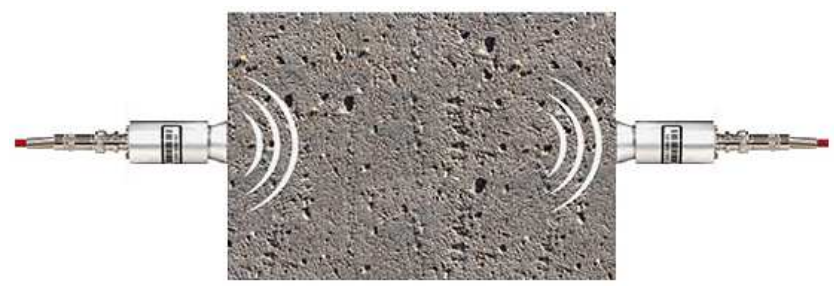

Figure 1. Plane ultrasonic transducers.

The detecting methods used most commonly are plane opposition detection, plane oblique detection and drilling detection. Plane opposition detection is applicable for the structure with a pair of plane surfaces for detection. Plane oblique detection is applicable for the structure with one surface for detection. Drilling detection is applicable for large scale structures. $[3,4]$
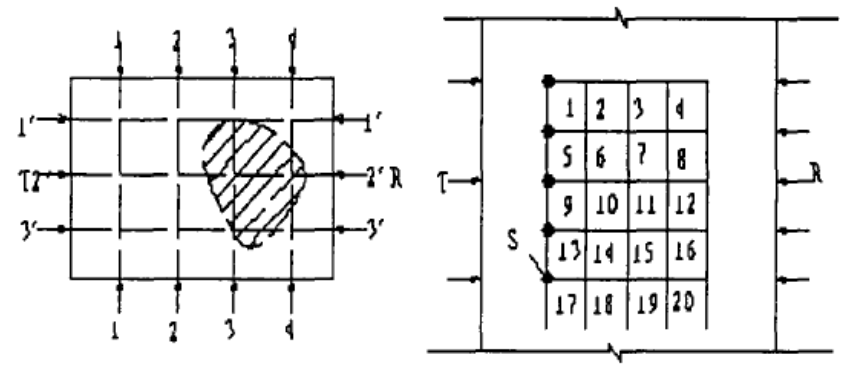

Figure 2. Plane opposition detection.

Different traditional ultrasonic detecting methods are applicable for various situations, but are all with limitations in operation due to the confirmed requirements for detection surface or testing distance. For instance, detecting surfaces of mass concrete or complicated structural concrete may not be convenient and appropriate for testing. If the thickness of tested structure is far beyond the effective transmission distance of ultrasonic transducer, the ultrasonic signal will be damped heavily [5-7]. Therefore, the data collected cannot support the analysis for concrete compactness. Additional, some locations of tested structure are difficult for technicians to achieve, like the edge area between the foundation and the reactor containment of nuclear power plant, where the space is too narrow for technician to perform detection.

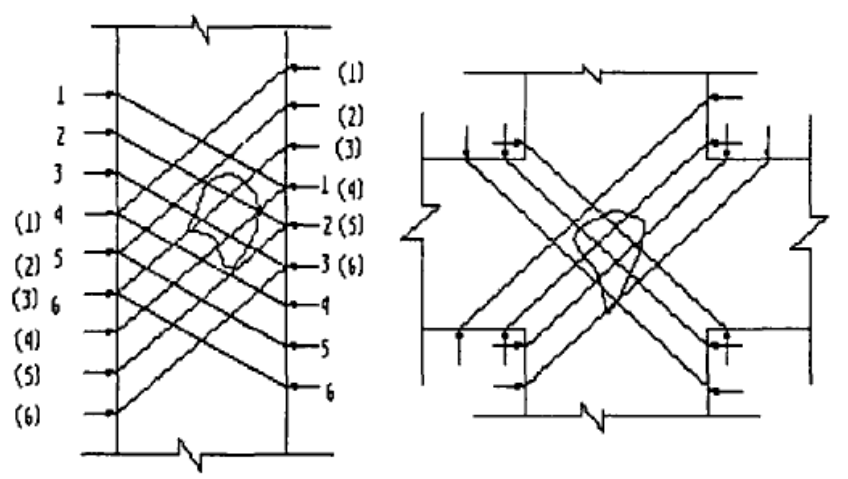

Figure 3. Plane oblique detection.

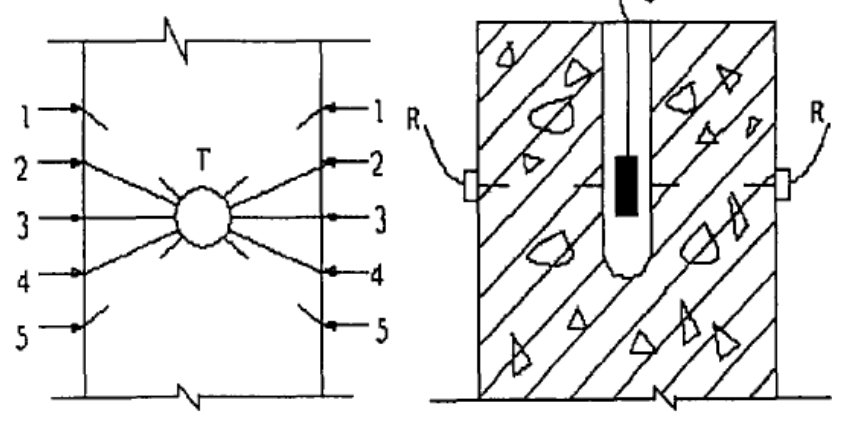

Figure 4. Drilling detection

\subsection{Impact-echo Detection}

Impact-echo method is another most common used approach for nondestructive detection. Impact-echo is suitable for the structure that only possesses one surface for detection, especially for thick element.[8] The method is performed on the base of transient stress (sound) waves. A small steel ball bearing is used as the impact source. By tapping the ball bearing ( $4 \mathrm{~mm}$ to $15 \mathrm{~mm}$ in diameter) against the structural surface an impact speed $(2 \mathrm{~m} / \mathrm{s}$ to $10 \mathrm{~m} / \mathrm{s})$, a short-duration (15 $\mu \mathrm{s}$ to $80 \mu \mathrm{s})$ mechanical impact will be produced and generate a low frequency stress wave. This wave propagates into the structure and is reflected by internal defects and external surfaces. An extremely sensitive boardbond transducer is located adjacent to the impact point to collect the reflected wave and transform the signal to the system monitor for analysis. 


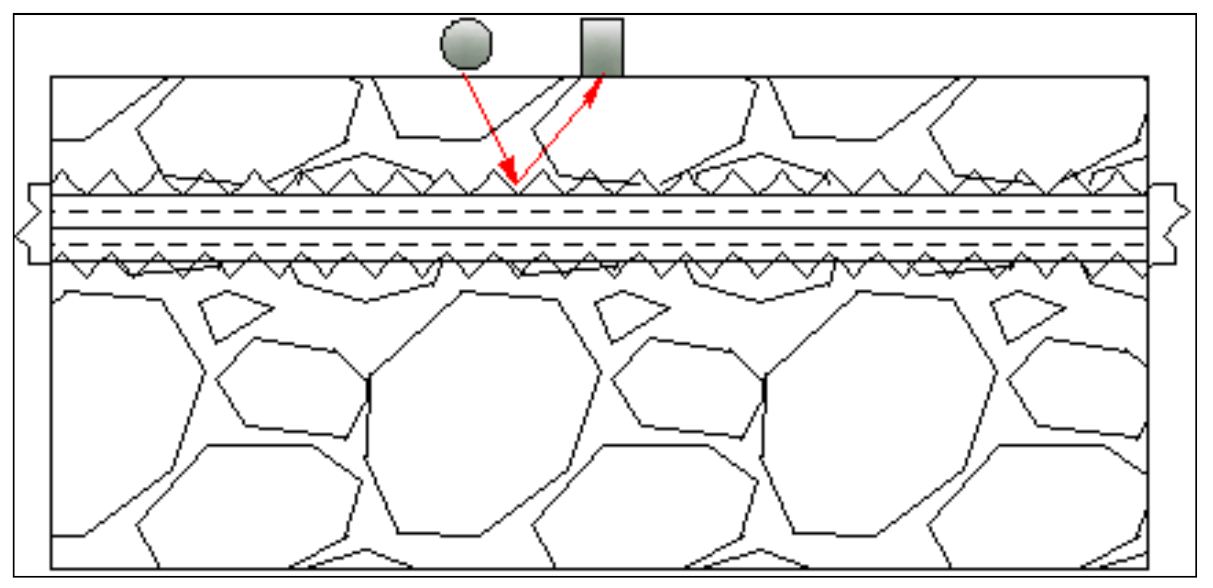

Figure 5. Impact-echo.

The impact-echo method is appropriate for testing concrete structures up to about $1.5 \mathrm{~m}$ thick as a result of the stress waves with correct range of frequencies and sufficient energy that generated by the impacts mentioned above.[9] Similar with ultrasonic detection, the effective testing range cannot fulfill the detection for large-scale structural elements. And the operation of impact-echo also demands an open area on the external surfaces of structures.

\section{Solutions}

On the basis of previous requirements, a spherical embedded ultrasonic transducer has been developed as per the structural characteristics of traditional ultrasonic transducer and the features of piezoelectric ceramics. This kind of transducer could be embedded inside the concrete structure during construction, and could motivate and receive ultrasonic waves from all directions inside structure. The transducer could be used pair with other spherical transducers embedded in array inside the structure, or with plane transducer handled by technician from the outside surface of structure to detect the compactness of mass and complicated concrete structures.

\subsection{Development of Spherical Embedded Ultrasonic Transducer}

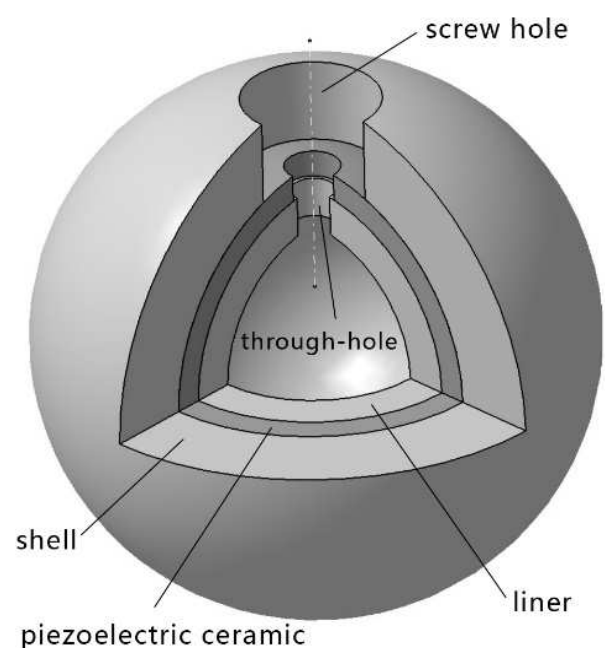

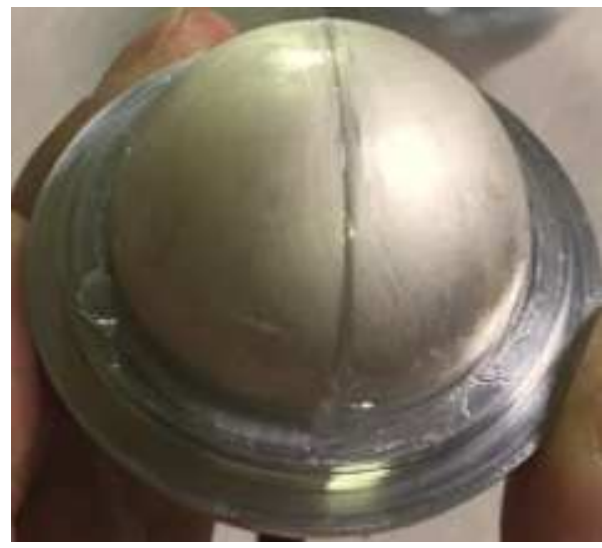

Figure 6. Design plan of spherical embedded ultrasonic transducer.

The structure and composition of spherical embedded ultrasonic transducer are illustrated in figure 6 . The transducer is composed of shell, piezoelectric ceramic crystal and liner. The material of piezoelectric ceramic is $\mathrm{PbTiO}_{3}-\mathrm{PbZrO}_{3}$ (PZT), which is extremely widely used in ultrasonic detection. PZT-52 is a specific type of production of PZT-5 series. PZT-52 is a receiving-type lead zirconate-titanate piezoelectric ceramic that possesses high electromechanical coupling factor, capacitivity, elastic constant and piezoelectric constant. PZT-52 possesses excellent time and temperature stability. Both of the resonance and non-resonance state could be used under low power. PZT-52 could be used to produce receiving-type transducer, transmit-receive transducer, or hydrophone. [10, 11]

The piezoelectric ceramic crystals are designed as two hemisphere shells assembled together. Both of the internal and external surfaces of shell are silver coating and polarized (figure 7). The liner and outer shell are also designed in two hemispheres. The outer shell, ceramic shell and liner of transducer are solidified together with adhesive like polyurethane. The through-hole on the top is used for the wire to pass through. A waterproof joint is arranged in the screw hole on the outer shell, which bottom is sealed with sealant to ensure its waterproof performance. The free end of wire is a $\mathrm{BNC}$ connector, which would be connected to the instrument during test. 


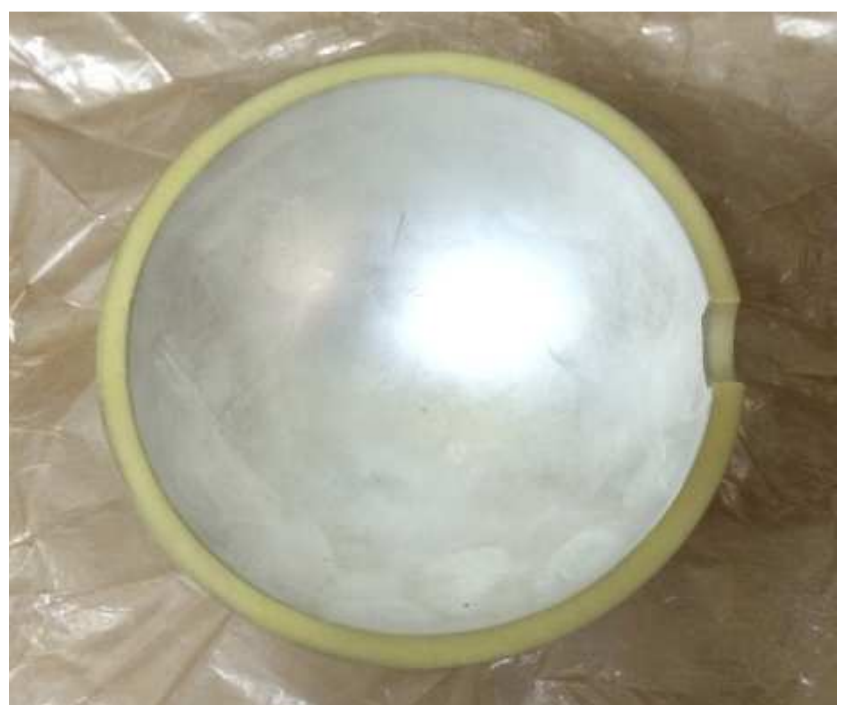

Figure 7. Hemisphere piezoelectric ceramic crystal.

\subsection{Application of Spherical Embedded Ultrasonic Transducer}

Figure 8 shows the spherical transducers adopted PZT-52 hemisphere piezoelectric ceramic crystals. Transducers of various sizes have been produced as per the different requirements. Different size of transducer adopts different size of piezoelectric ceramic crystal, which leads to different resonance frequency and different effective detecting distance (figure 9). The smaller the transducer is, the higher the frequency is, and the shorter the effective detecting distance is. Small-scale transducers are applicable for the high-accuracy demanded detection of small-scale structures. The bigger the transducer size is, the lower the frequency is, and the longer the detecting distance is. Big-scale transducers are suitable for the detection of large-scale structures.

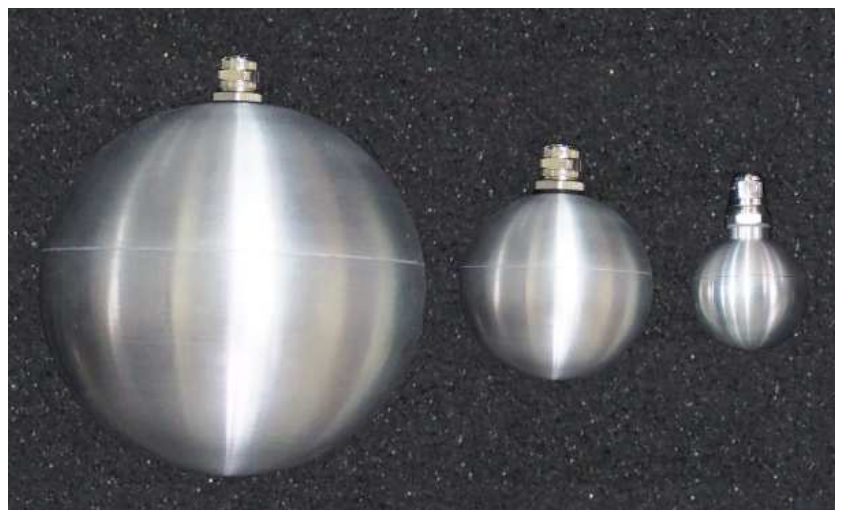

Figure 8. Spherical embedded ultrasonic transducer.

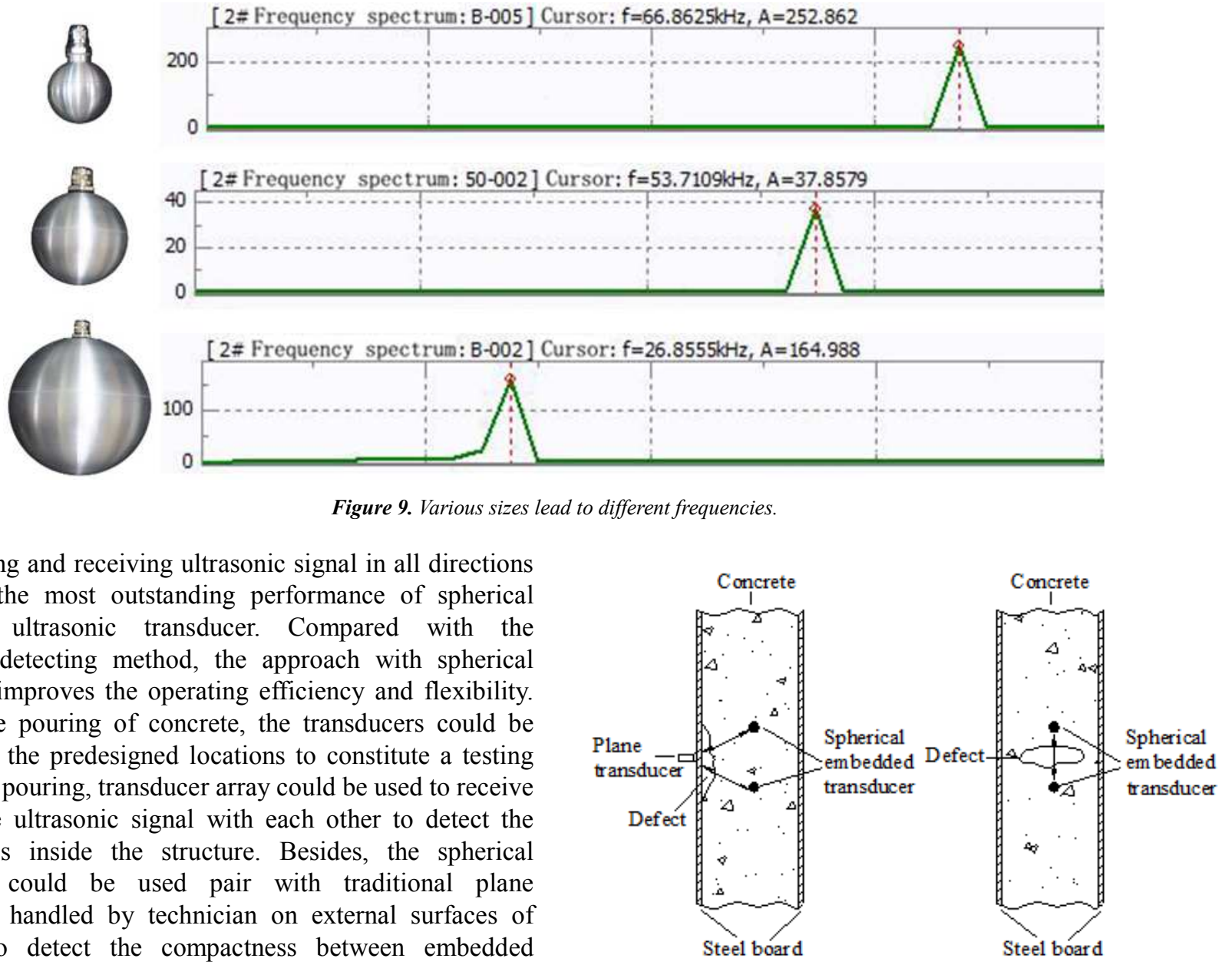

Figure 10. (L) Detection pair with plane transducer; (R) Detection with spherical transducers.

Motivating and receiving ultrasonic signal in all directions around is the most outstanding performance of spherical embedded ultrasonic transducer. Compared with the traditional detecting method, the approach with spherical transducer improves the operating efficiency and flexibility. Prior to the pouring of concrete, the transducers could be arranged at the predesigned locations to constitute a testing array. After pouring, transducer array could be used to receive or motivate ultrasonic signal with each other to detect the compactness inside the structure. Besides, the spherical transducer could be used pair with traditional plane transducers handled by technician on external surfaces of structure to detect the compactness between embedded transducer and outside transducer (figure $10 \& 11$ ). 

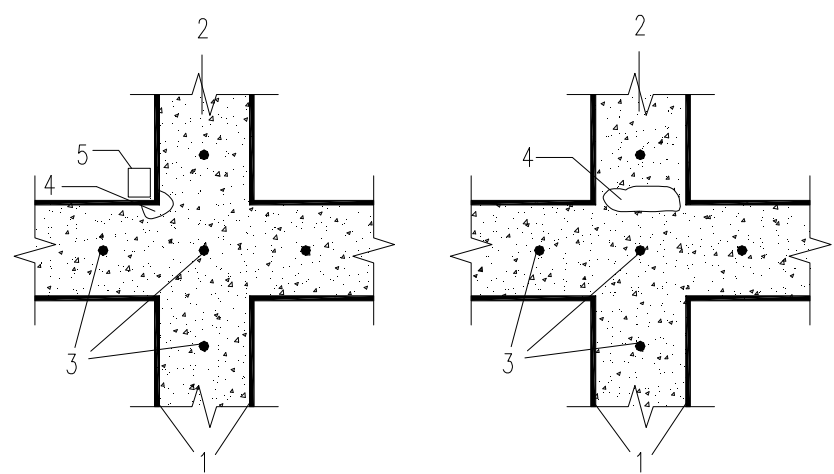

1-steel; 2-concrete; 3-spherical transducers; 4-defects; 5-plane transducer

Figure 11. Arrangement of spherical transducers at intersection position.

\subsection{Frequency Spectrum Analysis of Ultrasonic Signal}

A lot of information is included in the wave form of ultrasonic signal. Previously, most of ultrasonic detections always focus on the sound time, velocity and amplitude of ultrasonic wave, and estimate inside compactness situation of concrete structure in accordance with these parameters. However, these parameters are only parts of the wave form information. Therefore, the applicable range of ultrasonic has been limited by the estimating method mentioned previously. Additional, the testing results sometimes are not accurate and reliable enough due to the detection results would always be determined by the subjective experience of technician. The technique of frequency spectrum analysis has been applied widely in the material ultrasonic detection and evaluation. The compactness situation of specimen or structure could be judged more accurate and reliable by analyzing the frequency spectrum of ultrasonic signal.
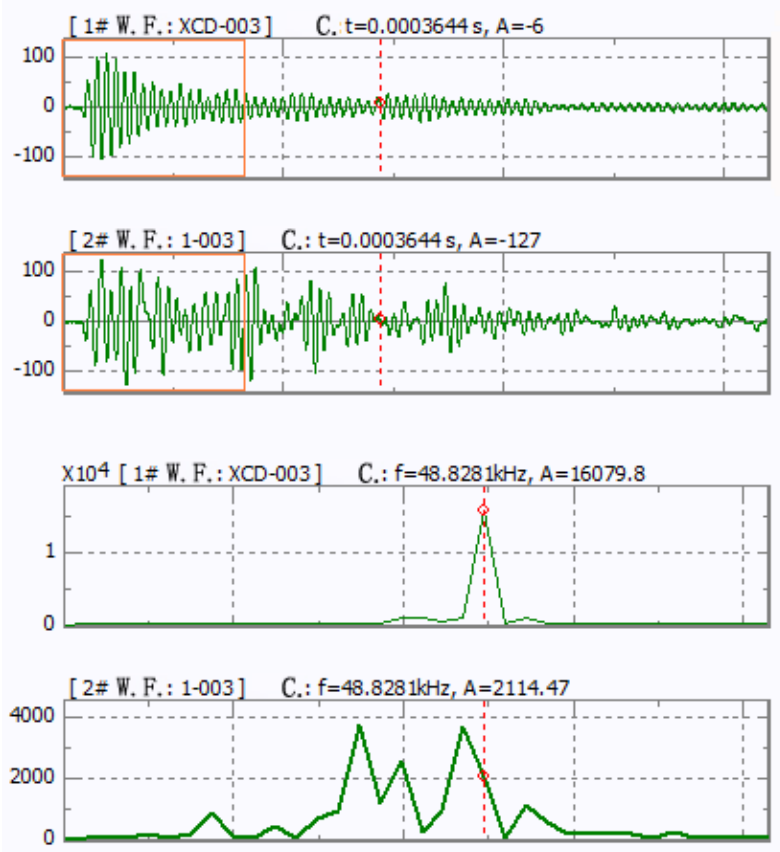

Figure 12. Frequency spectrum analysis of ultrasonic signal with software.

(Upper: Wave form of intact specimen; Below: Wave form of defect specimen; where W. F.: wave form; F. S.: frequency spectrum; C.: cursor)
Ultrasonic frequency spectrum analysis technique is a basic theory based on signal and system. The ultrasonic signal is as the input of the whole system that produced from the transducer by the motivation of pulse-generating circuit. The ultrasonic signal contains relavent information of detected specimen or structure after passing through them. The signal is received by another transducer and converted to the electric signal as the output of system. The output is subsequently transformed to frequency spectrum by Fast Fourier Transform (FFT). [12]

\section{Applications}

\subsection{Quality Supervision of Nuclear Power Plant Foundation}

The detecting method with spherical embedded ultrasonic transducer could be applied widely in the area of nuclear power plant, water conservancy project, traffic project, etc.

Take nuclear power plants for example, the structure between reactor containment and foundation is poured with Self-Compacting Concrete (SCC). SCC can flow freely by its own gravity, so that can completely fill the mold even if dense reinforcing bars are arranged inside the structure. The homogeneity of concrete can be ensured by the application of SCC. Additional, vibrating is unnecessary during pouring [13]. However, it is difficult to judge the compactness situation inside the structure after pouring, including the compactness of the position between concrete and the steel board at the bottom of containment. A solution adopted the spherical embedded ultrasonic transducer for this problem has been developed (figure 13). Arrange the spherical transducers at the predesigned position, subsequently supervise and detect the compactness situation of these positions with the embedded transducers and handled plane transducers.

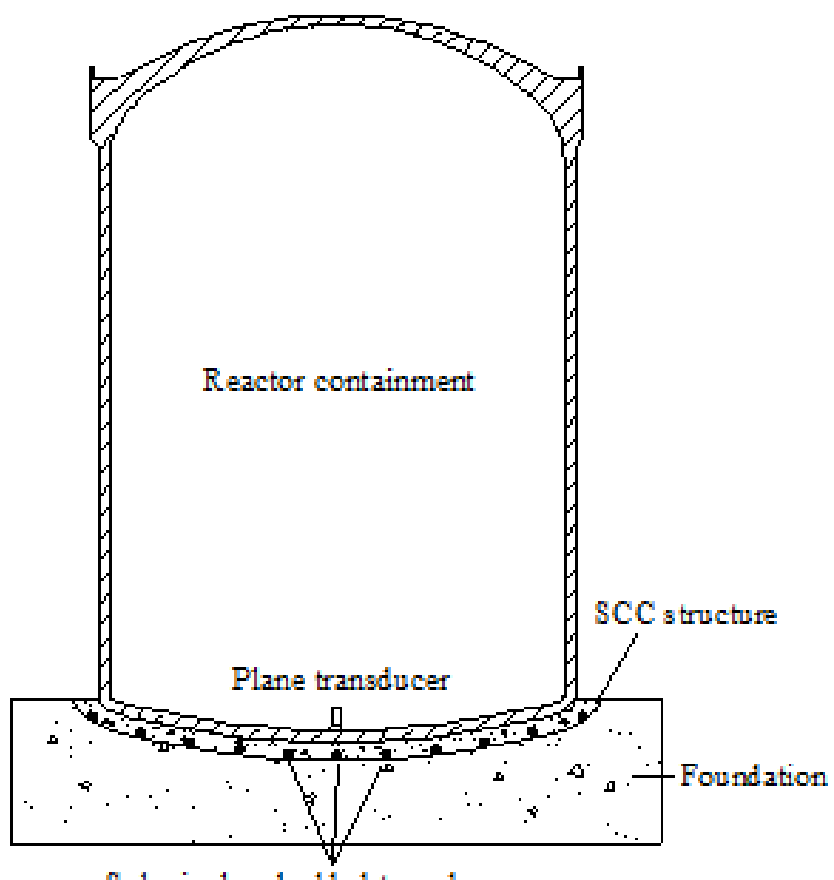

Spherical embedded transducer array 

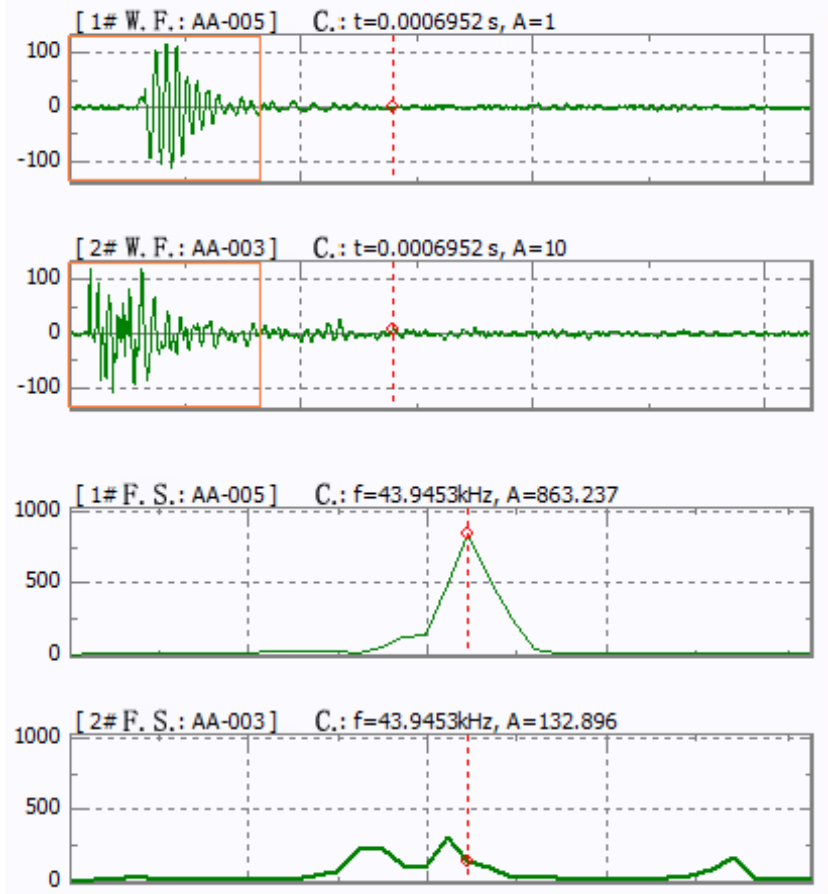

Figure 13. Technical proposal for concrete defect detection with spherical embedded ultrasonic transducer (L); wave form of normal position (up) vs defects (below) (R).

\subsection{Defect Detection of Other Structures}

The embedded transducer can also be applied in dam, rock slope, tunnel, etc. The method of application is similar with that applied in nuclear power plants. The specific technical proposal could be designed in accordance with different types of structures (figure 14).

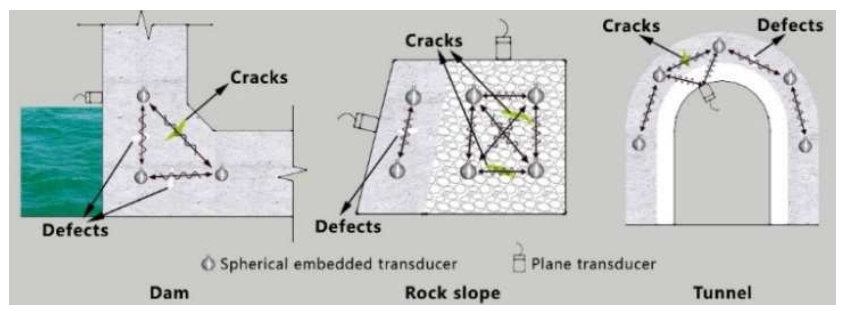

Figure 14. Embedded transducers applied in different structures.

\section{Conclusions}

Ultrasonic and impact-echo methods have been applied in concrete construction non-destructive detection. However, the limitations during application of these methods are obvious. When the detection is performed on the surfaces of structure, some defects not far from surfaces could be easily detected and located. But the effective transmission distance of ultrasonic is limited, so the defects deep inside the structure cannot be detected. The drilling ultrasonic detecting method mentioned previously allows the deep detection of structure to be achieved, but this method would damage the structural integrity. Impact-echo method could detect and locate the defects more accurately, but similar with ultrasonic surface detection, the transmission distance is limited and cannot be applied in mass concrete.

Compared with these methods, the detection with spherical embedded ultrasonic transducers could completely fulfill the requirements of detection for mass concrete and complicated structural elements. The spherical embedded ultrasonic transducer can motivate or receive ultrasonic signal pair with other spherical transducers by being arranged in some particular formation inside the structure, or with the traditional plane transducers handled by technician on the external surfaces of structure. This method could decompose a long detection distance into several short distances that could be achieved by ultrasonic signal effectively. Every two paired transducers inside structure are only used to detected or monitored the compactness situation of the structure between them.

The structural compactness can be analyzed by ultrasonic signal wave form and parameters. Similar with impact-echo, the frequency domain method can also be used to analyze the ultrasonic wave, and lead to a more accurate and reliable analyzing result

Further researches in the structure and production of spherical tranducers are still performing to improve the performances of thransducers. The method of concrete structure defects detection with spherical embedded ultrasonic transducers has been applied in nuclear power plants, and hopefully will be applied in other fields like irrigation projects and traffic projects in the future.

\section{Acknowledgements}

National Key Research and Development Program of "Development and Verification of Advanced Intelligent Aging Monitoring and Testing Technology for Important Equipment Components in Nuclear Power Plants" ( Project No. : 2020YFB1901500)

\section{References}

[1] Lin, C. G., Passive, safe and advanced nuclear power plant AP1000, Beijing: Atomic energy press, 2008.

[2] Dong, Q. H. "Progress of ultrasonic and acoustic detections for concrete," Concrete, Vol. 11, pp. 32-35, Nov. 2005.

[3] China Association for Engineering Construction Standardization, CECS 21:2000 Technical specification for inspection of concrete defects by ultrasonic method, Beijing: Standards press of China, 2001, pp. 8-9.

[4] Xie, J. "Research on the method of ultrasonic testing of concrete defects", Shanxi Architecture, Vol. 42, pp. 44-45, Dec. 2016.

[5] Zhu, J. L., Xiang, L. D., Liu, S. S., He, Y. M. \& Zhou, M. H. "Ultrasonic Sensor Application in Concrete Nondestructive Inspection System", Chinese Journal of Sensors and Actuators, Vol. 21, pp. 1290-1294, July 2008.

[6] Fang, L. L., "Research for the detecting methods of mass concrete", Technology Innovation and Application, Vol. 33, pp. 88-90, 2017. 
[7] Xiao, Y. Z., Yu, Y. B., Li, N., Hao, B. J., "Research for ultrasonic detection of the depth of concrete vertical fracture defect”, Shandong Indstrial Technology, Vol. 3, pp. 107-108, Feb. 2018.

[8] Li, Y. P., "On application of impact echo method in non-destructive detection of concrete buildings", Shanxi Architecture, Vol. 43, pp. 39-40, May. 2017.

[9] Sansalone, M., "Impact-Echo: The Complete Story", ACI Structural Journal, V. 94, No. 71, pp. 777-785, Nov.-Dec. 1997.

[10] Meng, Y. Q., "Research and Application of the Ultrasonic
Transducer", Journal of Shandong Polytechnic University, Vol. 26, No. 3, pp. 56-58, Aug. 2012.

[11] Luan, G. D., Zhang, J. D. \& Wang, R. Q., Piezoelectric transducers and arrays, 1st ed., Beijing: Peking university press, pp. 91-92, 2005.

[12] Lin, L \& Li, X. M., 1st ed., Ultrasonic frequency spectrum analysis technique and application, Beijing: China machine press, pp. 48, 2009.

[13] Guo, C. H. "Characteristics and applications of SCC," Science $\&$ technology information, Vol. 19, pp. 709, 2010. 